\title{
Resección hepática en el tratamiento de la litiasis intrahepática. Resultados inmediatos y a largo plazo en una serie occidental
}

\author{
C. Marín, R. Robles, P. Pastor y P. Parrilla \\ Departamento de Cirugía. Unidad de Cirugía Hepática y Trasplante Hepático. Hospital Universitario Virgen de la \\ Arrixaca. El Palmar, Murcia
}

\section{RESUMEN}

Objetivo: la litiasis intrahepática $(\mathrm{LIH})$ es una entidad poco frecuente en nuestro medio. Cuando se asocia a enfermedad de Caroli o a estenosis de los radicales biliares puede ser necesaria la resección hepática para su resolución definitiva. Presentamos los resultados a corto y largo plazo en una serie española de pacientes con resección hepática como tratamiento de la $\mathrm{LIH}$.

Pacientes: entre enero de 1996 y diciembre de 2007 realizamos una resección hepática en 8 pacientes por LIH. En 3 casos la LIH se asoció a enfermedad de Caroli y en los 5 casos restantes se asoció a estenosis de radicales segmentarios. La LIH se manifestó como colangitis aguda en 5 casos, como cólico hepático en dos casos y como pancreatitis recidivante en un caso. La técnica quirúrgica fue una hepatectomía derecha, 2 hepatectomías izquierdas y 5 resecciones segmentarias.

Resultados: no existió mortalidad intra- ni postoperatoria. La morbilidad fue del $25 \%$. En el seguimiento falleció un paciente (12\%) con enfermedad de Caroli malignizada a colangiocarcinoma. En los 7 pacientes restantes no ha habido recurrencia de la LIH tras un seguimiento medio de $62 \pm 2$ meses (rango 31-106).

Conclusiones: en nuestra experiencia, la resección hepática es el tratamiento indicado en aquellos pacientes con $\mathrm{LIH}$ asociada a estenosis y dilatación de la vía biliar, lobar o segmentaria, consiguiendo una resolución completa de la enfermedad con baja morbimortalidad.

Palabras clave: Litiasis intrahepática. Resección hepática. Colangiocarcinoma. Enfermedad de Caroli.

\begin{abstract}
Objective: intrahepatic lithiasis (IHL) is an uncommon entity in our environment. When associated with Caroli's disease or stenosis of the biliary radicals it may be necessary to perform liver resection to provide definitive resolution. We present immediate and long term results in a Spanish series of patients with hepatic resection to treat hepatolithiasis.

Patients: between January 1996 and December 2007 we performed a liver resection (LR) in 8 patients for IHL. The IHL was associated with Caroli's disease in 3 cases and with stenosis of segmentary radicals in the other 5 cases. It manifested itself as acute cholangitis in 5 cases, as biliary colic in two cases and recurrent pancreatitis in one case. The surgical technique was 1 right hepatectomy, 2 left hepatectomies and 5 segmentary resections.

Results: there was no intra- or postoperative mortality. The morbidity rate was $25 \%$. One patient $(12 \%)$ with Caroli's disease which had malignised to cholangiocarcinoma died in the follow-up period. The remaining 7 patients have had no IHL recurrence after a mean follow-up of $62 \pm 2$ months (range: 31-106).

Conclusions: in our experience liver resection, either lobar or segmentary, is the treatment for patients with IHL associated with stenosis and dilatation of the bile duct, as it provides complete resolution of the disease with low rates of morbidity and mortality.
\end{abstract}

Key words: Intrahepatic lithiasis. Liver resection. Cholangiocarcinoma. Caroli's disease.

Marín $C$, Robles $R$, Pastor $P$, Parrilla P. Resección hepática en el tratamiento de la litiasis intrahepática. Resultados inmediatos y a largo plazo en una serie occidental. Rev Esp Enferm Dig 2008; 100: 225-229.

\section{INTRODUCCIÓN}

La litiasis intrahepática (LIH), o presencia de cálculos en los conductos biliares intrahepáticos, es una entidad que

Recibido: 15-01-08.

Aceptado: 22-01-08

Correspondencia: Caridad Marín Hernández. Unidad de Trasplante Hepático. Hospital Universitario Virgen de la Arrixaca. 3ª Planta. 30120 El Palmar, Murcia. e-mail: rirocam@um.es afecta sobre todo a mujeres, siendo muy frecuente en países del Sudeste asiático (10-15\% de los pacientes sometidos a cirugía biliar por litiasis) (1-3) y menos frecuente en Sudamérica $(0,5-2 \%)$ y en Europa (4), existiendo pocas publicaciones en nuestro país y con un reducido número de casos (5-8). Esta patología puede asociarse a complicaciones como colangitis, abscesos hepáticos, insuficiencia hepática e incluso la malignización a colangiocarcinoma (CCC). Cuando los cálculos no se asocian a estenosis y se encuentran en los radicales biliares principales intrahepáticos, se 
Tabla I. Características generales de los 8 pacientes de nuestra serie

\begin{tabular}{|c|c|c|c|c|c|c|c|c|}
\hline & Caso 1 & Caso 2 & Caso 3 & Caso 4 & Caso 5 & Caso 6 & Caso 7 & Caso 8 \\
\hline Edad (años) & 44 & 34 & 45 & 45 & 58 & 29 & 38 & 77 \\
\hline Sexo & Varón & Varón & Varón & Varón & Varón & Mujer & Mujer & Varón \\
\hline Colecistectomía previa & No & No & No & Si (10 años antes) & Si (12 años antes) & No & No & No \\
\hline Clínica & Cólico hepático & Colangitis & Colangitis & Colangitis & Pancreatitis recidivante & Colangitis & Colangitis & Cólico hepático \\
\hline BT (mg/dl) & 1,6 & 1,5 & 0,8 & 2 & 8,6 & 6,7 & 5,3 & 2,3 \\
\hline GPT (u/l) & 35 & 125 & 40 & 89 & 102 & 86 & 96 & 240 \\
\hline $\mathrm{FA}(\mathrm{u} / \mathrm{l})$ & 325 & 350 & 125 & 300 & 401 & 310 & 287 & 301 \\
\hline $\mathrm{ECO}, \mathrm{TC}$ & Enf. Caroli, & Colelitiasis, & Colesterolosis, & Coledocolitiasis, & Enf. Caroli, & Colelitiasis, & Enf. Caroli, & Coledocolitiasis, \\
\hline RM preoperatoria & LIH & coledocolitiasis & absceso hepático & LIH & atrofia LIH & coledocolitiasis, LIH & microabscesos, LLH & LIH \\
\hline Localización & LHD & S. VI-VIII & S. V-VI & S. VIII & LHH & S. V-VIII & $L H$ & LHH \\
\hline CPRE preoperatoria & $\begin{array}{c}\text { Dilatación biliar } \\
\text { radicales Dx LH en LHD }\end{array}$ & No & No & $\begin{array}{l}\text { Coledocolitiasis, papilo- } \\
\text { tomía, extracción cálculos }\end{array}$ & $\begin{array}{c}\text { Amputación radical } \\
\text { biliar izada. }\end{array}$ & $\begin{array}{l}\text { Coledocolitiasis, papilo- } \\
\text { tomía, extracción cálculos }\end{array}$ & No & No \\
\hline CTPH & No & $\begin{array}{l}\text { LIH } \\
\text { S. VI-VIII }\end{array}$ & No & No & $\begin{array}{l}\text { Dilatación biliar } \\
\text { radicales izgda. }\end{array}$ & No & No & No \\
\hline
\end{tabular}

BT: bilirrubina total; CPTH: colangiotransparietohepática; LIH: litiasis intrahepática; LHD: lóbulo hepático derecho; LHI: lóbulo hepático izquierdo; TC: tomografía computerizada; RM: resonancia magnética; C-D: coledocoduodenostomía; S.: segmento hepático.

pueden extraer por coledocotomía, por vía endoscópica o por drenaje radiológico externo (9-11). En los casos en los que la LIH se asocia a estenosis o dilataciones de los radicales biliares intrahepáticos (enfermedad de Caroli) el tratamiento de elección es la resección hepática para evitar recurrencias y sus complicaciones $(12,13)$.

El objetivo de este artículo es presentar a 8 pacientes con LIH asociada a estenosis o dilataciones biliares en los que se realizó una resección hepática para su resolución definitiva, siendo la serie más larga recogida en la literatura española.

\section{PACIENTES}

Entre enero de 1996 y diciembre de 2007 se han realizado en nuestra unidad de cirugía hepática y trasplante hepático 475 resecciones hepáticas $(\mathrm{RH})$, siendo la indicación en 8 pacientes por LIH. La edad media fue de $42 \pm$ 3 años (rango: 29-77) con predominio de varones frente a las mujeres (6:2). Siete pacientes fueron de raza caucasiana y una mujer de raza oriental. El seguimiento medio de los pacientes ha sido de $62 \pm 2$ meses (rango: 31-106).

De los 8 pacientes, 2 habían sido colecistectomizados previamente (10 y 12 años antes). La clínica inicial fue de colangitis en 5 pacientes, cólico hepático en 2 pacientes y en el caso restante el debut de la enfermedad fue por una pancreatitis recidivante. En todos los casos hubo alteración de las enzimas hepáticas y la bilirrubina media fue de 3,6 g/dl (rango: 0,8-8,6). La ecografía preoperatoria fue la técnica de diagnóstico inicial en los 8 casos detectando la LIH en 6 pacientes, en 4 de ellos se apreció además una coledocolitiasis y en 3 casos la dilatación de los radicales biliares (enfermedad de Caroli). En los dos casos restantes, en los que no se detectó LIH, se apreciaron: en uno, abscesos en segmento V y VI; y en el otro, colelitiasis con coledocolitiasis. La tomografía computerizada (TC) y la resonancia magnética (RMN) confirmaron, en todos los casos, la pre- sencia de cálculos a nivel intrahepático. En 4 pacientes además se realizó una colangiopancreatografía endoscópica (CPRE) en la que se apreciaba LIH y dilataciones biliares en el lóbulo hepático derecho (LHD) en un caso, amputación del radical biliar izquierdo en otro y coledocolitiasis en los 2 casos restantes, que se extrajeron tras papilotomía. Dos pacientes fueron sometidos a colangiografía transparietohepática (CTPH) que demostró la LIH y estenosis del radical biliar del segmento VI en un caso y la enfermedad de Caroli en el lóbulo hepático izquierdo (LHI) en otro caso (Tabla I).

El abordaje terapéutico inicial en cinco pacientes fue una RH: una hepatectomía derecha (por enfermedad de Caroli asociada), 2 hepatectomías izquierdas (una de ellos asociada a enfermedad de Caroli), una resección del segmento VIII y una bisegmentectomía lateral izquierda (asociada a enfermedad de Caroli). En este último caso, se detectó en la anatomía patológica un CCC incidental, por lo que se reintervino para ampliar el margen quirúrgico realizando segmentectomía de los segmentos IV y I, resección de la vía biliar principal y reconstrucción en Y de Roux.

En los 3 pacientes restantes se realizó como primera intervención una colecistectomía y coledocoduodenostomía en 2 casos y una coledocotomía con cierre sobre un tubo en T en el tercer caso. En estos 3 pacientes la evolución fue tórpida presentando nuevos cuadros de colangitis por recidiva de la enfermedad por lo que se reintervinieron para realizar una RH como técnica definitiva (resección segmentaria VI y VIII; segmentectomía V y VI; y en el tercer caso una segmentectomía V y VIII) (Tabla II).

\section{RESULTADOS}

\section{Morbimortalidad precoz}

No hubo mortalidad postoperatoria. Ningún paciente precisó transfusión intra- ni postoperatoria. Dos pacientes (25\%) presentaron un absceso subfrénico con detección 
Tabla II. Técnica quirúrgica realizada en los 8 pacientes de nuestra serie

\begin{tabular}{|c|c|c|c|c|c|c|c|c|}
\hline & Caso 1 & Caso 2 & Caso 3 & Caso 4 & Caso 5 & Caso 6 & Caso 7 & Caso 8 \\
\hline $\begin{array}{l}1 \text { a intervención } \\
\text { Evolución } \\
\text { Colangio-transkehr }\end{array}$ & $\begin{array}{c}H D \\
\text { Curación }\end{array}$ & $\begin{array}{l}\text { Colecistectomía, } \\
\text { coledocotomía, CD } \\
\text { Colangitis } \\
\text { No }\end{array}$ & $\begin{array}{c}\text { Colecistectomía, } \\
\text { coledocotomía, tubo en T } \\
\text { Colangitis } \\
\text { LHH en S. V, VI }\end{array}$ & $\begin{array}{c}\text { Segmentectomía } \\
\text { VIII } \\
\text { Curación }\end{array}$ & $\begin{array}{c}\text { Bisegmentectomía } \\
\text { lateral izq. } \\
\text { AP: CCC }\end{array}$ & $\begin{array}{l}\text { Colecistectomía, } \\
\text { coledocotomía, CD } \\
\text { Colangitis } \\
\text { No }\end{array}$ & $\begin{array}{c}\text { Colecistectomía, } \\
\text { HI } \\
\text { Curación }\end{array}$ & $\begin{array}{c}\text { Colecistectomía, } \\
\text { Hl } \\
\text { Curación }\end{array}$ \\
\hline $2^{\mathrm{a}}$ intervención ECOI & & LIH en S. Vly VIII & LIH en S. Vy VI & & $\begin{array}{c}\text { Ampliación del } \\
\text { margen quirúrgico }\end{array}$ & LIHS. Vy VIII & & \\
\hline Técnica & & $\begin{array}{l}\text { Segmentectomía } \\
\text { VIy VIII }\end{array}$ & Segmentectomía V y VI & & $\begin{array}{l}\text { S IV-I. hepáticoyeyu- } \\
\text { nostomía en Y de Roux }\end{array}$ & $\begin{array}{c}\text { Segmentectomía } \\
\text { Vy VIII }\end{array}$ & & \\
\hline Evolución & & Curación & Curación & & $\begin{array}{l}\text { Recidiva hepática exitus } \\
\text { a los } 24 \text { meses }\end{array}$ & Curación & & \\
\hline
\end{tabular}

HD: hepatectomía derecha; CD: coledocoduodenostomía; LIH: litiasis intrahepática; S.: segmento; CCC: colangiocarcinoma; HI: hepatectomía izquierda.

en el cultivo de flora polimicrobiana, que se resolvieron con drenaje radiológico y antibióticos.

\section{Resultados a largo plazo}

Sólo el paciente en el que se encontró un CCC incidental falleció a los 24 meses de la intervención por recidiva hepática irresecable (mortalidad 12\%). En los 7 pacientes restantes no ha habido recurrencia de la LIH tras un seguimiento medio de $62 \pm 2$ meses (rango 31-106). Es de destacar que en los 5 pacientes en los que realizamos una RH como primera intervención no existió recidiva. Sin embargo, los 3 casos en los que se realizó sólo un abordaje de la vía biliar para extracción de cálculos presentaron nuevos cuadros de colangitis, por lo que precisaron de un RH para la resolución definitiva de la enfermedad.

\section{DISCUSIÓN}

La LIH puede constituir un problema grave, ya que presenta una morbilidad del $20-30 \%$ (a veces son necesarias numerosas reintervenciones para su resolución) y una mortalidad entre el 1-10\% (9-22).

El origen de los cálculos intrahepáticos puede ser doble: cálculos de formación intrahepática primaria y cálculos emigrados desde la vesícula al colédoco y de aquí a los radicales intrahepáticos (secundarios) (23). En el Sudeste asiático, la LIH se suele asociar a una estenosis de radicales biliares intrahepáticos que se cree secundaria a la infección por parásitos o bacterias, lo que condiciona una dilatación preestenótica, estancamiento de bilis con sobreinfección y formación primaria de cálculos (1220,23-25). En los países occidentales la LIH es poco frecuente y suele ser secundaria a un empedrado de la vía biliar principal y posterior migración de los cálculos al interior del hígado. Existen cálculos de formación primaria intrahepática $(12,15,26,27)$ debidos a: enfermedad de Caroli, colangitis esclerosante, colangiocarcinoma, estenosis biliares postraumáticas o postquirúrgicas, etc. En nuestra serie, los cálculos se formaron a nivel intrahepático de forma primaria en 3 casos, los cuales padecían una enfermedad de Caroli. Los 5 casos restantes fueron de origen secundario al asociarse a coledocolitiasis.

Se han descrito formas graves de presentación clínica $(12-21,26)$ (colangitis supurativa, hemobilia, abscesos hepáticos múltiples, cirrosis biliar secundaria con hipertensión portal y varices esofágicas, fallo hepático agudo e incluso colangiocarcinoma). En nuestra serie existieron cuadros recortados de colangitis aguda en cinco de nuestros pacientes que se controlaron fácilmente con tratamiento antibiótico y un paciente había desarrollado un colangiocarcinoma incidental, detectado en el estudio histológico y que falleció por recidiva tumoral a los 24 meses.

Desde el punto de vista diagnóstico, la ecografía preoperatoria es la técnica de imagen que debe hacer sospechar la presencia de LIH (5,27-30), siendo de utilidad para confirmar el diagnóstico la TC, la RMN y en especial la colangiorresonancia, que puede evitar la realización de pruebas invasivas. La CPRE tiene utilidad cuando existen cálculos en colédoco, ya que puede conseguir la extracción de los mismos (como ocurrió en dos de nuestros pacientes). En ocasiones permite visualizar las dilataciones biliares intrahepáticas y la LIH. Sin embargo, sus indicaciones deben ser individualizadas debido al riesgo de complicaciones infecciosas que asocia. La CPTH permite visualizar las dilataciones biliares, la LIH y la estenosis, como ocurrió en dos de nuestros pacientes. En tres de nuestros pacientes, la ECO y TC-RM sugirieron la presencia de LIH, aunque el cirujano no prestó importancia a este hallazgo, actuando sólo sobre la vesícula y el colédoco en una primera intervención, y ambos requirieron de una nueva intervención quirúrgica para resolver la LIH (Tabla II).

El tratamiento de la LIH puede ser quirúrgico o instrumental. Este último puede efectuarse bajo control radiológico percutáneo (colangioscopia transhepática percutánea, abordaje a través del tubo en T y otras) o endoscópico con o sin litroticia asociada $(3,12,16,29,31,32)$. El tratamiento quirúrgico de los cálculos se puede realizar a través de una 
coledocotomía, mediante hepatotomía o mediante resecciones hepáticas $(4,5-8,12,15,17,19,24,26,27,33-35)$.

Las indicaciones de una u otra forma de tratamiento dependen de las características de la LIH: aquellos cálculos secundarios a un empedrado coledociano, sin estenosis ni patología hepática subyacente, son con frecuencia fácilmente extraídos a través de la coledocotomía o mediante abordajes endoscópicos o radiológicos; sin embargo, en aquellos pacientes en los que la LIH se asocia a estenosis y dilataciones o bien a una patología hepática subyacente (Caroli, colangiocarcinoma, etc.) la simple extracción de los cálculos no es suficiente, ya que la recidiva es universal. En esta segunda situación, que es la que presentaron nuestros pacientes, la mayoría de autores $(5-8,12,15-19,26,29,32$ 38) considera a la resección hepática como la técnica de elección, ya que no sólo elimina la LIH y los factores responsables de su formación, sino que además elimina las posibles secuelas como un colangiocarcinoma asociado $(5-8,15,18,19,26,34)$, como ocurrió en uno de nuestros pacientes. Durante la intervención, la ECO intraoperatoria y la CIO son las exploraciones fundamentales para localizar los cálculos y asegurar que todos han sido eliminados $(28,30)$.

Las indicaciones de resección hepática son: lóbulo atrófico secundario a infecciones de repetición, cálculos limitados a un segmento o a un lóbulo y patología asociada (Caroli, colangiocarcinoma, estenosis, etc.). En la LIH bilateral, algunos autores $(11,17,24)$ obtienen buenos resultados con la realización de una hepatectomía izquierda y dilatación y extracción de los cálculos del lado derecho, reconstruyendo con una Y de Roux al hepático derecho, para posibles abordajes ulteriores $(36,38)$. En casos excepcionales y muy seleccionados en los que la resección hepática no es resolutiva, el trasplante podría plantearse como una opción terapéutica, sobre todo en aquellos pacientes en los que exista patología asociada que pueda malignizar, como es la enfermedad de Caroli (39). Cuando existe enfermedad de Caroli limitada al LHD o LHI, lo cual es poco frecuente, la hepatectomía derecha o izquierda es la técnica de elección, ya que en la actualidad dicha técnica se puede realizar con una baja morbimortalidad. Algunos autores (11-20) comunican que algunos pacientes requieren, tras la hepatectomía, un abordaje externo (radiológico y/o endoscópico) para extraer cálculos retenidos. En este sentido, algunos aconsejan $(2,11,12,15,36)$ asociar a estas técnicas derivaciones biliares a yeyuno asociadas a yeyunostomías para permitir futuras intervenciones endoscópicas $(16,36)$ o bien dejar un tubo en $\mathrm{T}$ en colédoco para posibles abordajes radiológicos externos posteriores (25).

En conclusión, en pacientes seleccionados con LIH asociada a estenosis o dilataciones segmentarias o lobares, donde fracasa la extracción radiológica o endoscópica, la RH es el tratamiento definitivo, siempre y cuando se realice con baja morbimortalidad.

\section{BIBLIOGRAFÍA}

1. Hwang JH, Yoon YB, Kim YT, Cheon JH. Risk factors for recurrent cholangitis after initial hepatolithiasis treatment. J Clin Gastroenterol 2004; 38 (4): 364-7.

2. Chen DW, Tung-Ping Poon R, Liu CL, Fan ST, Wong J. Immediate and long-term outcomes of hepatectomy for hepatolithiasis. Surgery 2004; 135 (4): 386-93.

3. Huang MH, Chen CH, Yang JC, Yang CC, Yeh YH, Chou DA, et al. Long-term outcome of percutaneous transhepatic cholangioscopic lithotomy for hepatolithiasis. Am J Gastroenterol 2003; 98 (12): 265562.

4. Catena M, Aldrighetti L, Finazzi R, Arzu G, Arru M, Pulitano C, et al. Treatment of non-endemic hepatolithiasis in a western country. The role of hepatic resection. Ann R Coll Surg Engl 2006; 88 (4): 383-9.

5. Ramia JM, Mansilla A, Villar J, García C, Garrote D, Ferrón JA. Enfermedad de Caroli monolobar asociada a hepatolitiasis. Cir Esp 2004; 75 (1): 46-7.

6. Robles R, Marín C, López J, Torralba JA, Lage A, Soria T, et al. Hepatectomía en 4 pacientes con litiasis intrahepática. Cir Esp 2002; 71 : $137-41$

7. Ramia JM, Palomeque A, Muffak K, Villar D, Ferrón JA. Indicaciones y opciones terapéuticas en la hepatolitiasis. Rev Esp Enferm Dig 2006; 98 (8): 597-604.

8. Manzanera M, Hidalgo M, Hernández D, Jiménez C, Rico P, Gimeno A, et al. Surgical treatment of hepatolithiasis. Presentation of a case. Rev Esp Enferm Dig 1998; 90: 51-2.

9. Cheung MT, Wai SH, Kwok PC. Percutaneus transparietohepatic choledochoscopic removal of intrahepatic stones. Br J Surg 2003; 90 (11): 1409-15.

10. Kim YT, Byun JS, Kim J, Jang YH, Lee WJ, Ryu JK, et al. Factors predicting concurrent cholangiocarcinomas associate to hepatolithiasis. Hepatogastroenterology 2003; 50 (49): 8-12.

11. Uchiyama K, Onishi H, Tani M, Kinoshita H, Ueno M, Yamaue H. Indication and procedure for treatment of hepatolithiasis. Arch Surg 2002; 137 (2): 149-53.

12. Otani K, Shimizu S, Chijiwa K, Ogawa T, Monsaki T, Sugitani A, et al. Comparison of treatments for hepatolithiasis: Hepatic resection versus cholangioscopic lithotomy. J Am Coll Surg 1999; 189: 177-82.

13. Fan ST, Lai E, Mok FP, Choi TK, Wong J. Acute cholangitis secondary to hepatolithiasis. Arch Surg 1991; 126: 1027-31.

14. Jeng, KS, Ohta I, Yang FS, Liu TP, Shih SC, Chang W, et al. Coexisting sharp ductal angulation with intrahepatic biliary strictures in right hepatolithiasis. Arch Surg 1994; 129: 1097-102.

15. Do KS, Tran GK, Doan TT, Nguyen TG, Do MH, Do T, et al. Hépatectomie dans la lithiase intrahépatique. Chirurgie 1999; 124: 626-31.

16. Maetani I, Ishiguro J, Ogawa S, Sato M, Igarashi Y, Sakai Y. Percutaneous choledochoscopic treatment of intrahepatic stone including management of associated biliary stenoses. Endoscopy 1999; 31: 456-9.

17. Jeng KS, Ohta I, Yang FS. Reappraisal of the systematic management of complicated hepatolithiasis with bilateral intrahepatic biliary strictures. Arch Surg 1996; 131: 141-7.

18. Chen MF, Jan YY, Hwang TL, Jeng LB, Yeh TS. Impact of concomitant hepatolithiasis on patients with peripheral cholangiocarcinoma. Dig Dis Sci 2000; 45: 312-6.

19. Chang TM, Passaro E. Intrahepatic stone: The Taiwan experience. Am J Surg 1983; 146: 241-4.

20. Yeh TS, Tseng JH, Chen TC, Liu Nj, Chiu CT, et al. Characterizacion of intrahepatic cholangiocarcinoma of the intraductal growth-type and its precursor lesions. Hepatology 2005; 42 (3): 657-64.

21. Guma C, Viola C, Apestegui M, Thome U, Tani D, Kido N, et al. Hepatolitiasis y enfermedad de Caroli en Argentina: resultados de un estudio multicéntrico. Acta Gastroenterol Latinoam 1999; 29: 9-15.

22. Di Carlo I, Sauvanet A, Belghiti J. Intrahepatic lithiasis: A Western experience. Surg Today 2000; 30: 319-22.

23. Takada T, Uchiyama K, Yasuda H, Hasegawa H. Indications for the choledochoscopic removal of intrahepatic stones based on the biliary anatomy. Am J Surg 1996; 171: 558-61.

24. Chen MF, Jan YY, Wang, CS, Hwang TL, Jeng LB, Cheu CS. Role of hepatic resection in surgery for bilateral intrahepatic stones. Br J Surg 1997; 84: 1229-32. 
25. Cheung MT. Postoperative choledochoscopic removal of intrahepatic stones via tube tract. Br J Surg 1997; 84: 1224-8.

26. Lee TY, Chen YL, Chang HC, Chan CP, Kuo SJ. Outcomes of hepatectomy for hepatolithiasis. World J Surg 2007; 31: 479-82.

27. Azuma T, Yoshikawa T, Araida T, Takasaki K. The significance of hepatectomy for primary intrahepatic stones. Surg Today 1999; 29: 1004-10.

28. Liu CL, Fan ST, Wong J. Primary biliary stones: Diagnosis and management. World J Surg 1998; 22: 1162-6.

29. Gillet M, Favre S, Fontolliet C, Halkic N, Mantion G, Heyd B. Maladie de Caroli monolobaire. À propos de 12 cas. Chirurgie 1999; 124: 13-8.

30. Zhang W, Niu HO, Zhao GW, Su KJ, Wei HC, Su ZX, et al. Use of intraoperative ultrasonography during hepatolithectomy. World J Surg 1996; 20: 50-4.

31. Jeng KS, Sheen IS, Yang FS. Are expandable metallic stents better than conventional methods for treating difficult intrahepatic biliary strictures with recurrent hepatolithiasis? Arch Surg 1999; 134: 267-73.

32. Adamek H, Schneider A, Adamek M, Jakobs R, Buttmann A, Benz C, et al. Treatment of difficult intrahepatic stones by using extracorporeal and intracorporeal lithotripsy techniques: 10 years experience in 55 patients. Scand J Gastroenterol 1999; 34: 1157-61.

33. Adson M, Nagorney D. Hepatic resection for intrahepatic ductal stones. Ach Surg 1982; 117: 611-6.

34. Lee SK, Seo DW, Myung SJ, Park ET, Lim BC, Kim HJ, et al. Percutaneous transhepatic cholangioscopic treatment for hepatolithiasis: An evaluation of long-term results and risk factors for recurrence. Gastrointest Endos 2001; 53: 318-23.

35. Yoshida J, Chijiiwa K, Shimizu S, Sato H, Tanaka M. Hepatolithiasis: Outcome of cholangioscopic lithotomy and dilation of bile duct stricture. Surgery 1998; 123: 421-6.

36. Fan ST, Mok F, Zheng SS, Lai E, Lo CM, Wong J. Appraisal of hepaticocutaneous jejunostomy in the management of hepatolithiasis. Am J Surg 1993; 165: 332-5.

37. Li SQ, Liang LJ, Peng BG, Lu MD, Lai JM, Li DM. Bile leakage after hepatectomy for hepatolithiasis: Risk factors and management. Surgery 2007; 141: 340-5.

38. Cheung MT, Kwok PC. Liver resection for intrahepatic liver stones. Arch Surg 2005; 140 (10): 993-7.

39. Balsells J, Margarit C, Murio E, Lázaro JL, Charco R, Vidal MT, et al. Adenocarcinoma in Caroli's disease treated by liver transplantation. HPB Surg 1993; 7 (1): 81-6. 